\title{
Paraplegia due to Missed Thoracic Meningioma after Laminotomy for Lumbar Spinal Stenosis: Report of Two Cases
}

\author{
Sang-Bong Ko, Sang-Wook Lee, Jung-Hyun Shim \\ Department of Orthopaedic Surgery, Daegu Catholic University Medical Center, \\ Catholic University of Daegu School of Medicine, Daegu, Korea
}

\begin{abstract}
To describe two cases of thoracic paraplegia due to a thoracic spinal cord tumor (meningioma) that was not detected during lumbar spinal decompressive surgery for lumbar canal stenosis and a complaint of claudication. The follow-up period ranged from 1 year and 6 months to 1 year and 8 months. The neurological deficit due to thoracic meningioma after surgery for lumbar canal stensois was decreased after mass excision. So, careful physical examination and magnetic resonance imaging can reveal another thoracic spine compressive lesion such as meningioma. Additional thoracic decompressive surgery can provide partial amelioration of each patient's neurological condition. Surgeons should know that a silent meningioma can aggrevate neurological symptoms after lower lumbar spine surgery and should inform their patient before surgery.
\end{abstract}

Key Words: Thoracic paraplegia, Meningioma, Lumbar surgery

\section{Introduction}

Among many complications resulting from spinal surgery [1,2], the most painful and dreaded complication is neurological deterioration [2], especially motor weakness. Reports on neurological deterioration due to a missed compressive lesion in the spinal cord above the level targeted for lumbar decompression surgery are very rare [1-3]. Boccanera and Laus [1] reported three cases, Valls et al. [2] reported one case, and Takeuchi et al. [3] reported three cases. The cases of Boccanera and Laus [1] were cases of neurological deterioration after decompressive surgery for lumbar spinal stenosis. The case of Valls et al. [2] were cases of thoracic paraparesis after routine laminectomy. The cases of Takeuchi et al. [3] were cases of thoracic paraplegia after decompressive surgery. In all seven cases, the neurological deterioration was successfully ameliorated by additional decompressive surgery.
In this report, we describe two cases of thoracic paraplegia due to a thoracic spinal cord tumor (meningioma) that was missed on routine preoperative imaging as well as on the preoperative physical examination since an upper lesion was not suspected during the initial lumbar spinal decompressive surgery for lumbar spinal stenosis.

\section{Case Reports}

\section{Case 1}

This was a case of 73-year-old woman who suffered from low-back pain, an insidious onset of bilateral neurogenic claudication (she was limited to a $50 \mathrm{~m}$ walking distance), and lower-extremity motor weakness (right and left tibialis anterior, Grades 3 and 4, respectively; right and left extensor hallucis longus, Grades 3 and 4, respectively; right gastrocnemius, Grade 3). The patellar and Achilles tendon reflexes were hypotonic. An magnetic resonance imaging

Received Jun 16, 2010; 1st Revised Jul 9, 2010; 2nd Revised Oct 1, 2010; Accepted Oct 1, 2010

Corresponding author: Sang-Bong Ko, MD

Department of Orthopaedic Surgery, Daegu Catholic University Medical Center,

3056-6 Daemyeong 4-dong, Nam-gu, Daegu 705-718, Korea

Tel: +82-53-650-4283, Fax: +82-53-626-4272, E-mail: bong@cu.ac.kr 
(MRI) revealed stenotic changes at a lower level (L4-5-S1) (Fig. 1). Preoperative post-myelographic radiographs and computed tomography $(\mathrm{CT})$ revealed stenotic changes at the lower level but did not include any levels above T6 in the views examined. Decompressive surgery was performed at L4-5-S1 by a spinal orthopaedic surgeon (SBK). On the 14th postoperative day, the patient exhibited lower-extremity motor weakness bilaterally (right and left iliopsoas, Grades 2 and 3, respectively; bilateral quadriceps, Grade 2; right and left tibialis anterior, Grades 2 and 3, respectively; bilateral extensor hallucis longus, Grade 2; and bilateral gastrocnemius, Grade 2). The patient refused the surgeon's readmission recommendation. Two weeks after the onset of symptoms, she was readmitted to our hospital. Her bilateral patellar tendon reflexes were exaggerated and the anal sphincter tone and the lower extremity motor was insidiously weakened. Thoracic MRI revealed canal compression from T3-4 (Fig. 2), and an intradural extramedullary tumor was suspected. A second operation was performed to excise the tumor one month after the 1st operation. A histopatho-
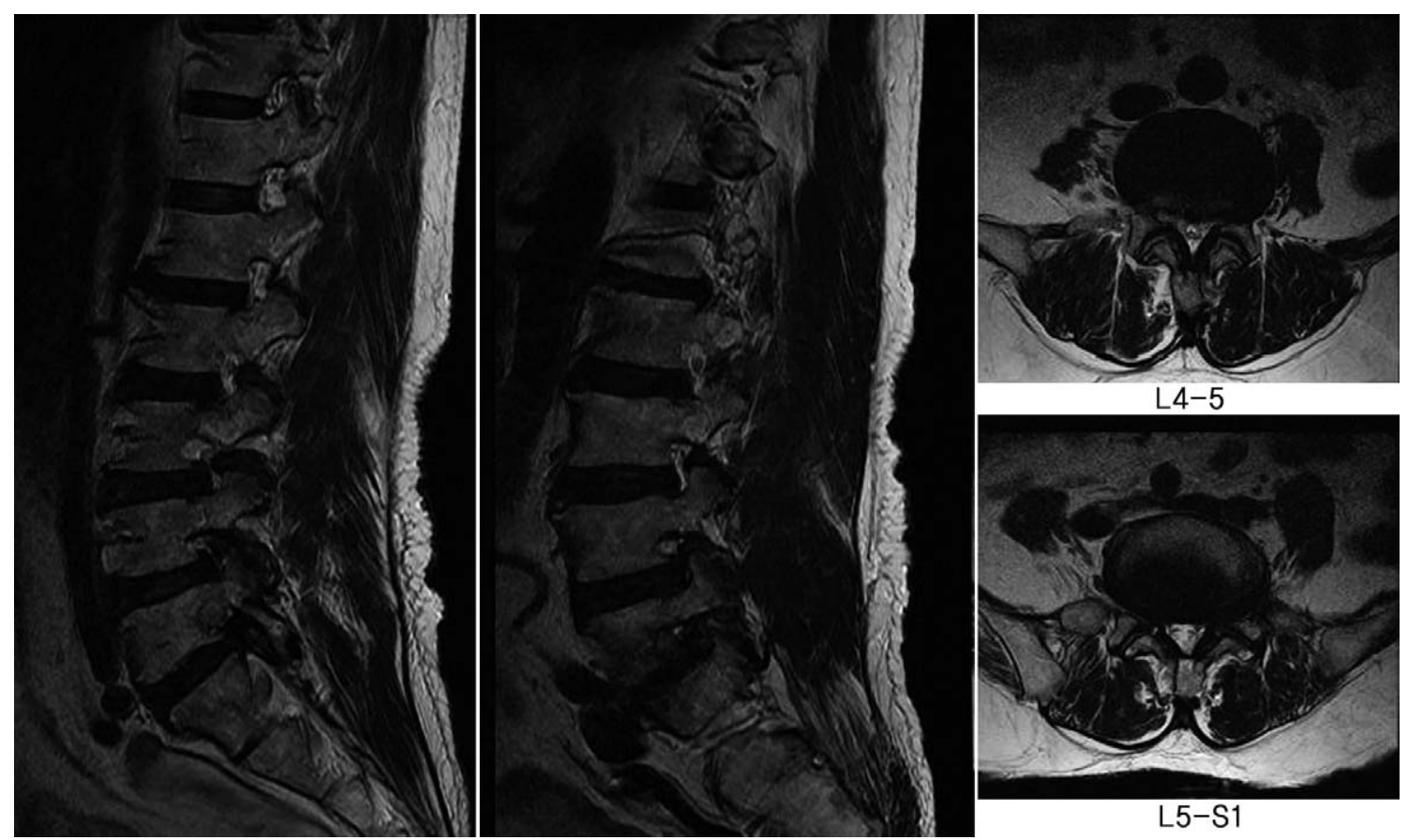

Fig. 1. Magnetic resonance imaging findings of lumbar spine showed both foraminal stenosis, L4-5-S1 due to disc herniation, degenerative hypertrophic changes of the both facet joints.
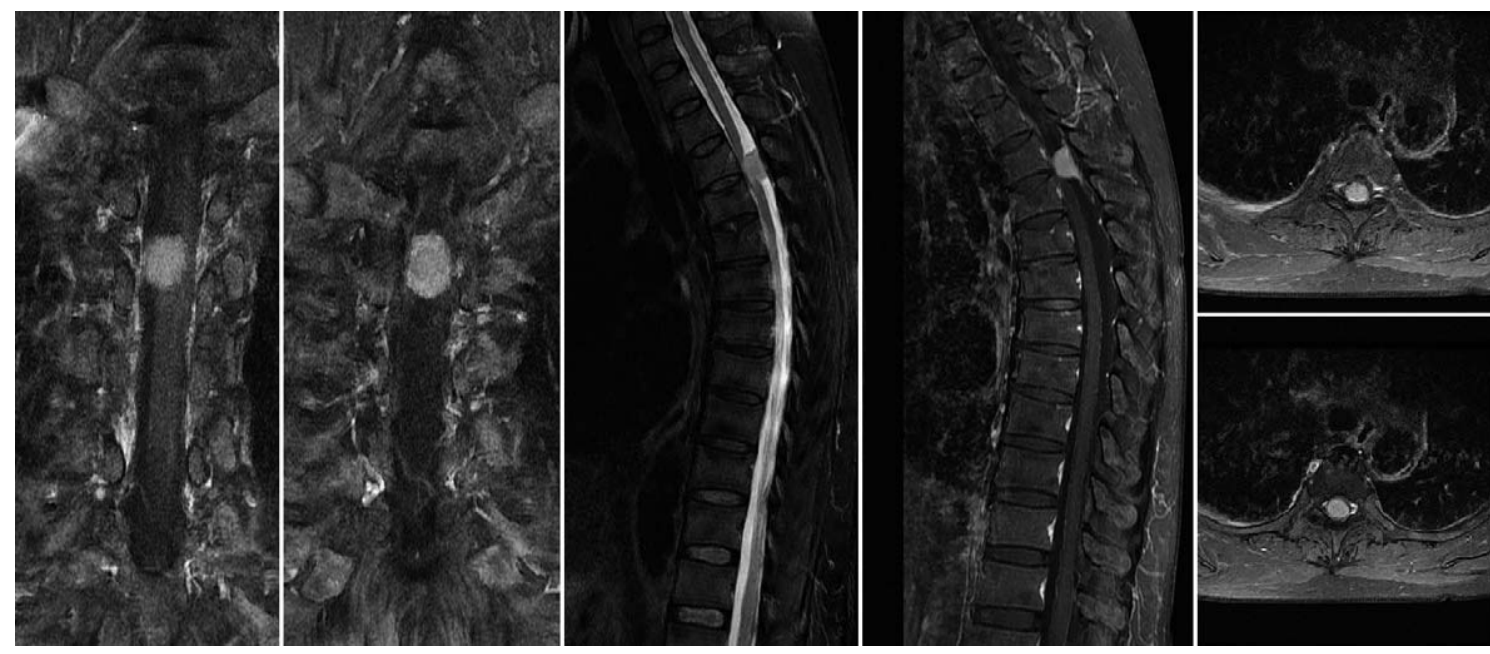

Fig. 2. Magnetic resonance imaging findings of thoracic spine showed $1.6 \mathrm{~cm}$-sized extramedullary intradural mass; suspicious meningioma at T3-4 level. 
logical examination showed this tumor to be a Grade 1 meningioma. One year and eight months after the second surgery, her neurological status improved, including urinary function, anal tone, and motor function (bilateral iliopsoas, quadriceps, tibialis anterior and extensor hallucis longus Grade 5; right and left gastrocnemius, Grades 4 and 5, respectively).

\section{Case 2}

This was a case of a 63-year-old woman who suffered from low-back pain, bilateral neurogenic claudication (limited to a walking distance of $30 \mathrm{~m}$ ), and lower-extremity motor weakness (bilateral iliopsoas, Grade 5; bilateral quadriceps, Grade 5; right and left tibialis anterior, Grades 4 and 5; bilateral extensor hallucis longus, Grade 3; bilateral gastrocnemius, Grade 4). The deep patellar and Achilles tendon reflexes were absent. MRI revealed stenotic changes at L3-4-5 levels (Fig. 3). Preoperative postmyelographic radiographs and $\mathrm{CT}$ revealed no abnormal findings below the T5 level. Decompressive surgery was performed at L3$4-5$ by a spinal orthopaedic surgeon (SBK). On the 3rd postoperative day, the patient exhibited lower-extremity motor weakness bilaterally (bilateral iliopsoas, Grade 2; bilateral quadriceps, Grade 2; bilateral tibialis anterior, Grade 1; bilateral extensor hallucis longus, Grade 1; right and left gastrocnemius, Grades 1 and 0, respectively). Bilateral patellar and Achilles tendon reflexes were exaggerated and anal sphincter tone was weakened. Sensory changes were seen at the 4th thoracic spinal level. Thoracic MRI revealed a $2.7 \times 1.3 \mathrm{~cm}$ broad-based, strongly enhancing mass in the intradural space of T3-5 (Fig. 4). A second operation was performed to excise the tumor seven days after the 1st operation. One year and six months after the second surgery, her neurological status improved, including urinary function, anal tone, and motor function (right and left iliopsoas, Grades 4 and 5 respectively; right and left quadriceps, Grades 4 and 5 respectively; right and left tibialis anterior, Grades 4 and 5 respectively; bilateral extensor hallucis longus and gastrocnemius, Grade 4).

\section{Discussion}

In these two cases, the thoracic spine was examined via postmyelographic radiographs and CT but the imaging was limited to levels below the T6 level. After lumbar decompressive surgery, neurological deterioration occurred between days 3 and 14. After a thorough physical examination, severe thoracic compressive lesions were detected on thoracic spine MRI, which prompted a second decompressive surgery at the level above the first surgery to resolve neurological deficits.

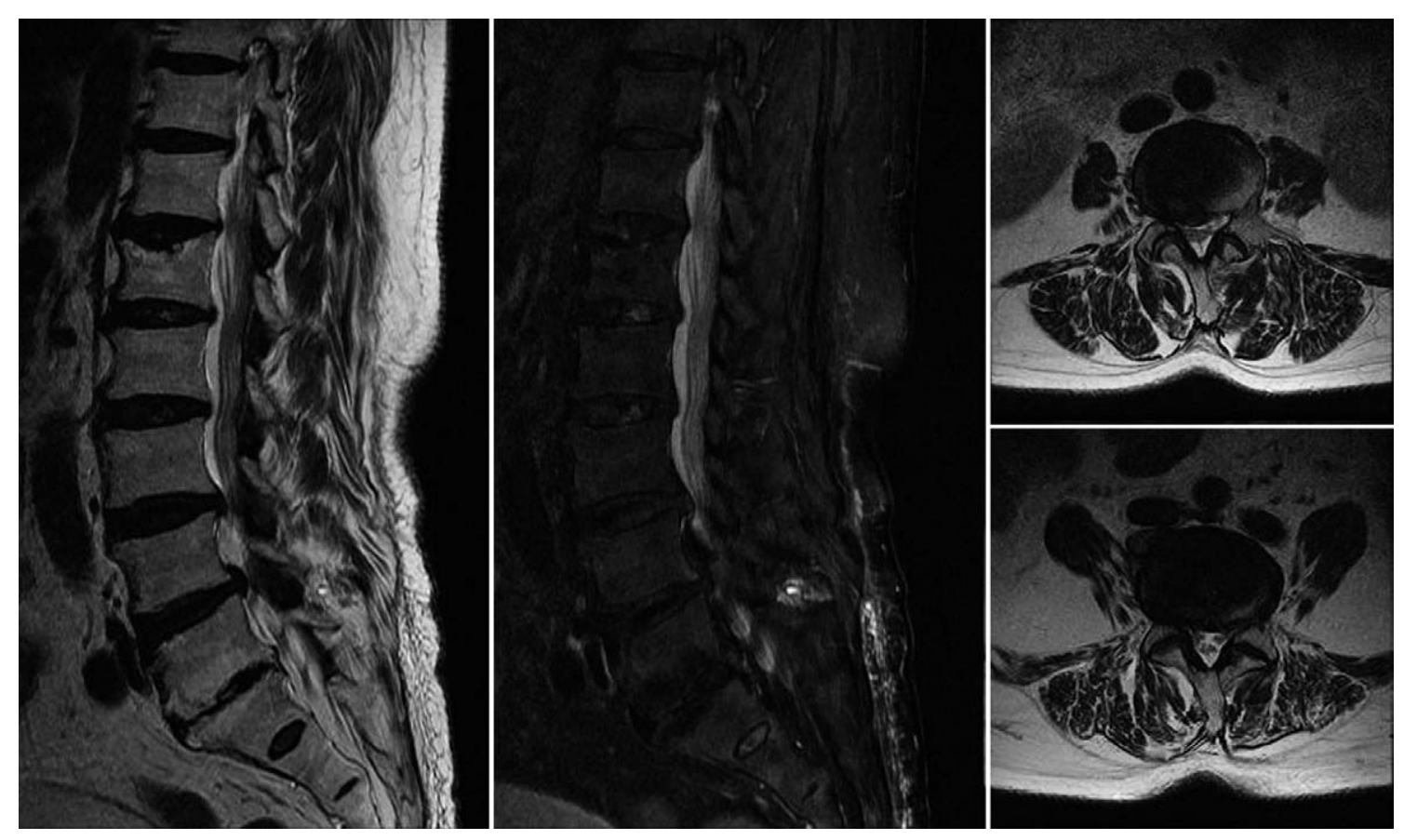

Fig. 3. Magnetic resonance imaging findings of lumbar spine showed right foraminal stenosis, L3-4-5 due to herniated disc. 


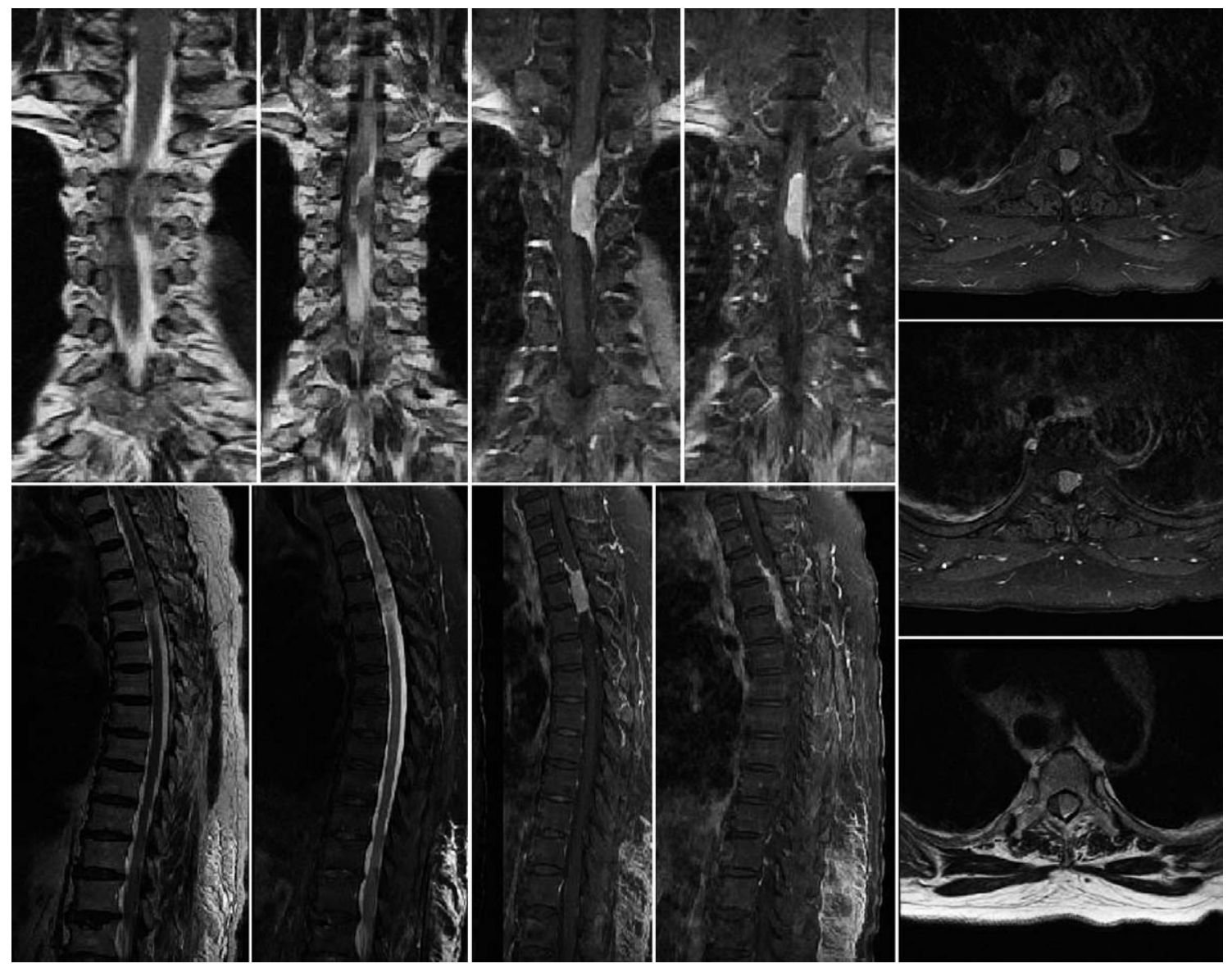

Fig. 4. Magnetic resonance imaging findings of thoracic spine showed $2.7 \times 1.3 \mathrm{~cm}$ sized strong enhancing mass with dural tail sign in intradural space of T3-5 level; suspicious meningioma.

Common complications such as hematoma, nerve root injury [4] and cauda equina syndrome [5] at the operative site have been reported. However, only rarely has a deteriorating neurologic symptom been reported as a result of a coexisting lesion above the level of the operative site [1-3].

Spinal meningiomas are located in the intradural extramedullary space, grow slowly, spread laterally in the subarachnoid space, and are usually symptomatic - motor deficit (61.8\%) at 6.2 months [6]. Woltman et al. [7] found that the mean delay in diagnosis of intradural extramedullary tumors was 2.9 years. The symptoms of a typical thoracic meningioma are secondary to an insidious course of gradual tumor growth and cord compression.

The cause of the neurological deterioration at the level above the operative site was posulated to be due to dynamic changes in cerebrospinal fluid flow. However, this has not been proven. Boccanera and Laus [1] suggested that the pressure changes due to previous decompressive surgery constricted the neural elements and caused deterioration in neurological status at the level of the preexisting missed compression. Valls et al. [2] discussed the idea that dynamic changes in cerebrospinal fluid flow due to the initial operation may influence the missed thoracic arachnoid cyst and lead to worsening of neurologic symptoms. However, Turker et al. [8] reported that the cause of the neurological deterioration was not due to preexisting compression and instability but rather to spinal cord infarction and cord edema. Takeuchi et al. [3] also suggested that the effect of the initial lumbar decompressive surgery on the flow dynamics of cerebrospinal fluid may cause the thoracic compressive lesions to become symptomatic. All these postulated mechanisms aggravate the meningioma induced neurological symptoms acutely. However, the exact pathophysiological mechanism could not be determined.

Approximately $80 \%$ of spinal meningiomas are located in the thoracic spine; fewer in the cervical spine (15\%), lumbar spine (3\%), and the foramen magnum (2\%). Most intradural spinal tumors are benign and potentially resectable, so the prognosis after surgical resection is excellent $[9,10]$. 
Careful neurological examination including sensory, motor and autonomous function tests can help avoid and minimize such a misdiagnosis. If only we had paid more attention to the fact that in the patients with compressionrelated thoracic myelopathic symptoms and compressionrelated lumbar symptoms, the long tract signs, such as exaggerated deep tendon reflexes and other upper motor neuron signs of the lower extremities, tend to be masked. Then we would have decided not to push through with the lumbar spine decompressive operation on the basis of an inconclusive lumbar spine MRI and lower extremity motor weakness. Thus, if lumbar spine imaging findings do not correctly correspond to the patient's complaints or the results of the neurological examination, the spinal surgeon, we suggest, should perform a thorough neurological examination for thoracic or cervical lesions such as pinprick testing of the trunk or upper extremity, and then check the upper level MRI before doing the lumbar spine operation.

In these two cases, recovery of good neurological status was partially due to the onset of symptoms after the first and second operations. A long duration between the two operations would have brought about irreversible spinal cord damage. However, evidence for this hypothesis is unavailable.

To provide a hypothesis for changes in the dynamics of cerebrospinal fluid flow after decompressive procedures, greater clinical experience will be needed. Thoracic paraplegia occurred in these two cases because compressive thoracic lesions were not detected during initial lumbar decompressive surgery. To avoid such a complication, we suggest that examination of the thoracic spine should be performed preoperatively if lumbar imaging is inconclusive or is incompatible with the history and physical examination.

\section{REFERENCES}

1. Boccanera L, Laus M. Cauda equina syndrome following lumbar spinal stenosis surgery. Spine (Phila $\mathrm{Pa} 1976$ ) 1987;12:712-5.

2. Valls PL, Naul LG, Kanter SL. Paraplegia after a routine lumbar laminectomy: report of a rare complication and successful management. Neurosurgery 1990;27:638-40.

3. Takeuchi A, Miyamoto K, Hosoe H, Shimizu K. Thoracic paraplegia due to missed thoracic compressive lesions after lumbar spinal decompression surgery: report of three cases. J Neurosurg 2004;100(1 Suppl Spine):71-4.

4. Bertrand G. The "battered" root problem. Orthop Clin North Am 1975;6:305-10.

5. Kostuik JP, Harrington I, Alexander D, Rand W, Evans D. Cauda equina syndrome and lumbar disc herniation. J Bone Joint Surg Am 1986;68:386-91.

6. Gelabert-González M, García-Allut A, Martínez-Rumbo R. Spinal meningiomas. Neurocirugia (Astur) 2006;17:12531.

7. Woltman HW, Kernohan JW, Adson AW, Craig WM. Intramedullary tumors of spinal cord and gliomas of intradural portion of filum terminale: fate of patients who have these tumors. AMA Arch Neurol Psychiatry 1951;65: 378-95.

8. Turker RJ, Slack C, Regan Q. Thoracic paraplegia after lumbar spinal surgery. J Spinal Disord 1995;8:195-200.

9. Frank BL, Harrop JS, Hanna A, Ratliff J. Cervical extradural meningioma: case report and literature review. J Spinal Cord Med 2008;31:302-5.

10. Salehpour F, Zeinali A, Vahedi P, Halimi M. A rare case of intramedullary cervical spinal cord meningioma and review of the literature. Spinal Cord 2008;46:648-50. 